\title{
Comparison of Post Exposure Effect of Music through Personal Listening Device (PLD) on Hearing in Young Adults
}

\author{
Hrudananda Sahoo* \\ Mysore University, India
}

Submission: September 6, 2016; Published: September 20, 2016

*Corresponding author: Hrudananda Sahoo, Mysore University, At-Beparibrahampur, Po/Dist- Jajpur, Odisha, India, Tel: 9408246832, Email: hrudanigam@gmail.com

\begin{abstract}
With the increasing massive use of Personal Listening Devices (PLDs) among young individuals, the major concern is that excessive exposure to high sound pressure levels (SPLs) may lead to a temporary or permanent hearing loss in this population. The purpose of the present study is to measure the output SPL of the PLDs at three different condition (quiet, bus noise and at maximum volume control setting) using probe microphone measurement. Also to evaluate auditory measures including high frequency audiometry, transient evoked otoacoustis emissions and speech perception in noise before and after abstaining from music for a period of 15 days. Results indicated that measured output SPL in the presence of bus noise and at the maximum volume control setting of the devices were higher than the quite condition. None of the auditory measures shows significant improvement before and after getting abstained from music. In general it is suggestive of permanent damage to both peripheral as well as central auditory structure.
\end{abstract}

Keywords: Personal listening devices (PLDs); High sound pressure level; Young adults

Abbreviations: TEOAEs: Transient Evoked Otoacoustic Emissions; SNR: Signal-to-Noise Ratio; PLDs: Personal Listening Devices; SPLs: Sound Pressure Levels

\section{Introduction}

The leading preventable cause of acquired sensorineural hearing loss is exposure to excessive levels of noise, which leads to irreversible loss of cochlear hair cells. But of late, due to massive growth in the popularity of personal MP3 players, young adults are exposed to high levels of mu-sic, and they may be at risk for permanent hearing loss every time they listen to their favorite music. Most often it is seen that the people prefer using personal listening devices (PLDs) during travelling or be-fore sleeping. The rapid development of digital technology has produced new kinds of PLDs whose sound quality at higher volumes is much better, because the sound is no longer distorted. Because the music players are equipped with improved earphones, sound leakage is almost absent, which means that the music players can be played at hazardous high volumes in most environments without disturbing others.

Hearing loss induced by PLDs may evolve into a significant social and public health problem in future years. Previous investigations have revealed that output sound pressure levels produced by personal music systems are in 80-120 dBA range. Preferred listening levels were slightly higher for ear bud style of earphones compared to the over-the-ear style [1]. In addition, ear bud type which gives a tailor-made fit in ear canal in-creases the problem by direct channeling the sound into the ear [2]. The output levels of the PLDs depend upon the type of PLDs, listening environment and style of headphone [1-6]. Kumar et al. [6] evaluated the mean output SPLs at preferred listening settings in quiet, in the presence of $65 \mathrm{~dB}$ SPL bus noise and at maximum volume control settings for mobile phones, iPods and locally made MP3 players.

The mean loudness equivalent exposure for continuous 8-hour of duration (Leq $8 \mathrm{hr}$ ) were $73 \mathrm{dBA}$ for mobile phones (Range: $40 \mathrm{dBA}$ to $93 \mathrm{dBA}$ ), $76 \mathrm{dBA}$ for iPods (Range: $56 \mathrm{dBA}$ to $86 \mathrm{dBA}$ ), and $79 \mathrm{dBA}$ for locally made MP3 players (Range: $70 \mathrm{dBA}$ to $84 \mathrm{dBA}$ ), at subject preferred volume control settings in quiet. Listening in the presence of bus noise did not increase the output SPLs significantly but at the maximum volume control settings output levels in-creased compared to the subject preferred volume control setting. It has been shown in the previous studies that the use of PLDs may have hazardous effect on hearing [6,7]. Tao et al. [7] reported that the hearing thresholds in the $3 \mathrm{kHz}, 4 \mathrm{kHz}, 6 \mathrm{kHz}$, and $8 \mathrm{kHz}$ frequency range were significantly increased in PLDs listener though hearing 
thresholds in low frequencies were within normal range. Kumar et al. [6] also reported that listening to music through PLDs at a preferred volume control setting may not result in "clinically significant" elevation of hearing threshold and may not be evident during routine pure-tone audiometry. Furthermore, Kumar et al. [6] reported that amplitude of high frequencies otoacoustic emissions were reduced in individuals who listen to high level of music.

\section{Methods \\ Participants}

58 Participants in the age range of 16-26 years (mean age of 22 years, 23 males and 35 females) participated in the present research. During selecting participants the body mass index and the other factor were taken care. These participants were divided into two groups. The Group-I consisted of 29 participants who report as the regular user of personal listening devices (PLDs) and Group-II consisted of 29 participants, served as the controls, who were not regular users of PLDs. All the Group-I users used to prefer to listening music during their daily travelling which is around 3-4 hour per day. Participants in both the groups had their air conduction and bone conduction hearing threshold within $15 \mathrm{~dB}$ HL at octave frequencies from $250 \mathrm{~Hz}$ to $8 \mathrm{kHz}$. All participants showed 'A' type tympanogram with acoustic reflex at normal sensation levels. None of them reported any history of middle ear pathology, ototoxic drugs usage, exposure to occupational noise or not reported any habit of smoking and drinking.

\section{Procedure}

The study was conducted in three phases. In the first phase output sound pressure level were measured. In the second phase hearing threshold, speech perception in noise and otoacoustic emissions were measured. In the third phase auditory measurements were repeated after asking PLD users to abstain listening to music for 15 days to evaluate the nature of hearing deficits, if any, being temporary or permanent.

\section{Phase I- Measurement of output sound pres-sure levels (SPL) of PLDs}

Using a probe microphone, the output SPLs produced by PLDs were measured in the participant's ear canal. Fonix -7000 was used for this purpose. The probe microphone insertion depth of $28 \mathrm{~mm}$ (tip of the tube to the tragal notch) was maintained for all participants. All measurement were carried out with the subjects own PLDs and earphones. The earphone was placed after placing the probe tube in the ear canal. Before the measurement was done subjects were asked to play one of their favorite songs. Output SPLs were measured in three different conditions:

a. In quite - the subject was asked to set the volume control to their usual preferred listening setting. In the presence of $65 \mathrm{~dB}$ SPL bus noise, the subject was asked to set the volume control to their preferred listening setting. Bus noise was given through a personal laptop. Bus noise was considered as back-ground noise as this condition is more naturalistic since most of the participants listen to music while commuting. At the maximum volume control settings of the instrument. Position of the probe microphone remained constant in all measurement conditions.

b. Diffuse field SPLs to which ear were exposed was calculated by subtracting the transfer function of the open ear from the obtained ear canal SPL. This transformation is required to compare the output of PLDs to damage risk criteria. Hence, the ear canal sound pressure levels will be converted into to diffuse field levels by subtracting the transfer function of the open ear canal.

c. The transfer function of the open ear was measured by calculating the difference between the probe microphone SPLs near the eardrum for a sweep frequency tone presented at $65 \mathrm{~dB}$ SPL and reference location which is at the opening of the ear canal. The output SPLs at each frequency was converted to dBA values by adding the A weighting adjustment values. The overall SPL in ABA was calculated by adding the octave band levels logarithmically.

\section{Phase II- Auditory measures}

In this Phase extended high frequency audiometry, otoacoustic emissions, and speech perception in noise were assessed. Extended high frequency audiometry Calibrated two channel diagnostic audiometer GSI 61 with transducer HDA 200 was used for extended high frequency audiometry. Using modified version of Hughson and Westlake procedure [8] pure tone hearing thresholds were estimated at different frequencies from $3 \mathrm{kHz}$ to $20 \mathrm{kHz}$. Otoacoustics emission Transient evoked otoacoustic emissions (TEOAEs) were recorded using commercially available otoacoustic emission analyzer (ILO-V6). Subjects were asked to sit on a reclining chair. TEOAE probe was inserted into their ear canal and TEOAEs were measured for $80 \mathrm{~dB}$ peak SPL clicks. Average response from a total of 260 non linear clicks was used for the analysis. The overall TEOAE amplitudes and amplitudes at $1000 \mathrm{~Hz}, 1414 \mathrm{~Hz}, 2000 \mathrm{~Hz}, 2828$ $\mathrm{Hz}$ and $4000 \mathrm{~Hz}$ frequency bands were noted and used for analysis.

Speech perception in noise in the present study, speech intelligibility was measured using a signal-to-noise ratio (SNR) required for $50 \%$ identification using the sentence list developed by Methi \& Kumar [9]. Seven equivalent lists from the original test were selected for the present study. Each list contained 7 sentences mixed with the eight talker speech babble noise at a different signal to noise ratios (SNRs). First sentence in each list was at $+8 \mathrm{~dB} S N R$, second sentence was at $+5 \mathrm{~dB}$ SNR, third sentence was at $+2 \mathrm{~dB}$ SNR, fourth sentence was at $-1 \mathrm{~dB}$ SNR, fifth sentence was at - 4dB SNR, sixth sentence was at -7dB SNR and last sentence was at $-10 \mathrm{~dB}$ SNR. Each sentence had 5 key 
words. These sentences were presented through a personal computer at comfortable levels using a commercially available headphone. The listener's task was to repeat the sentences presented and each correctly repeated key word was awarded one point for a total possible score of 35 points per list.

\section{Phase III- Re-evaluation of the auditory measures}

The same test protocol that is extended high frequency audiometry, otoacoustic emissions, and speech perception in noise were repeated again after a gap of 15 days on PLD user group with the assurance that the participants had abstained from listening to music using PLD. Hereafter for easy nomenclature the Groups-I is named as PLDs-users, the Group-II who had given a 15 days of rest period will be named as PLDsrest and the Group-III being named as Non-user.

\section{Results}

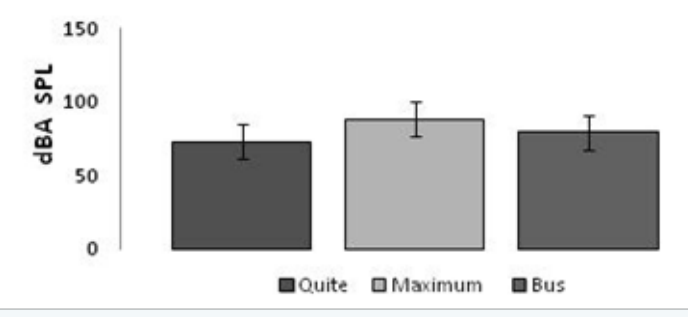

Figure 1: Mean output sound pressure level in quiet and in the presence of $65 \mathrm{~dB}$ SPL bus noise, and maximum volume control settings of the instrument. Error bars show 1 standard deviation.

Measurement of output sound pressure levels (SPL) of personal listening devices (PLDs) (Figure 1) show the average output levels in quiet condition, in presence of $65 \mathrm{~dB}$ SPL bus noise and the maximum output limits of the devices along with one standard deviation of error. The average output levels in quiet con-dition was $73.34 \mathrm{dBA}$, at maximum output levels of the devices is at $88.36 \mathrm{dBA}$ and in presence of $65 \mathrm{~dB}$ SPL bus noise was $79.44 \mathrm{dBA}$. Paired t-test was performed to see the significance of difference between mean overall SPL between three conditions. Results showed that mean over all SPLs were significantly higher in bus noise $(\mathrm{t}=-3.134, \mathrm{p}<0.05)$ and at maximum volume control settings $(t=-6.297, p<0.05)$ of the instrument compared to quiet condition.

\section{Auditory measure}

Extended High Frequency measures (Figure 2) and (Figure 3) shows the average high frequency threshold of the PLDuser, PLD users after the rest period of 15 days (called PLD-rest hereafter) and Non-PLD user group for the right ear and (Figure 2c) and (Figure 2d) shows similar information for left ear. From (Figures 2-5) it can be inferred that hearing thresholds in the high frequency region were poor in PLD user group compared to non user group. Since standard deviations of the high frequency thresholds were high non parametric test was used to evaluate the significance of difference among hearing thresholds in PLD user, PLD rest and PLD non user group.

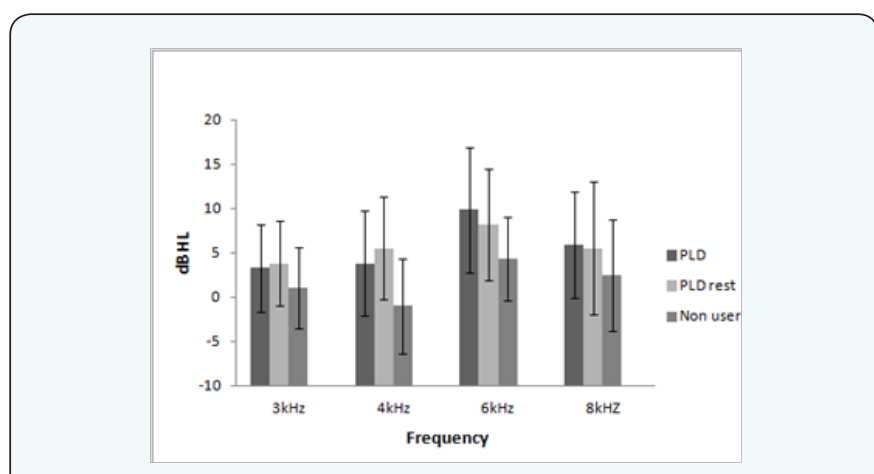

Figure 2: The mean high frequency hearing threshold across 3 $\mathrm{kHz}$ to $8 \mathrm{kHz}$ frequencies in the right ear for PLD-user, PLD-rest and Non-user.

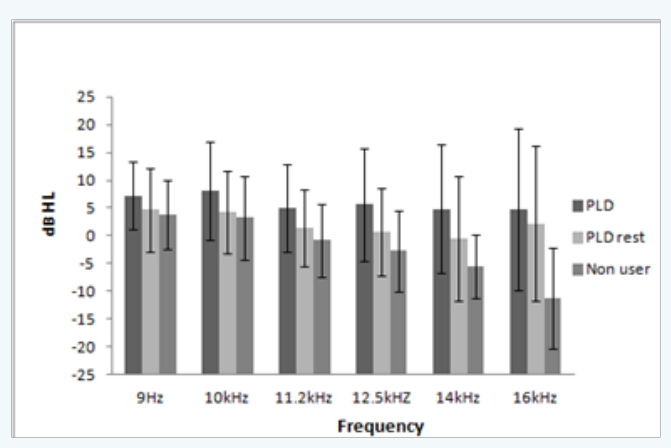

Figure 3: The mean high frequency hearing threshold across 9 $\mathrm{kHz}$ to $16 \mathrm{kHz}$ frequencies in the right ear for PLD-user, PLD-rest and Non-user.

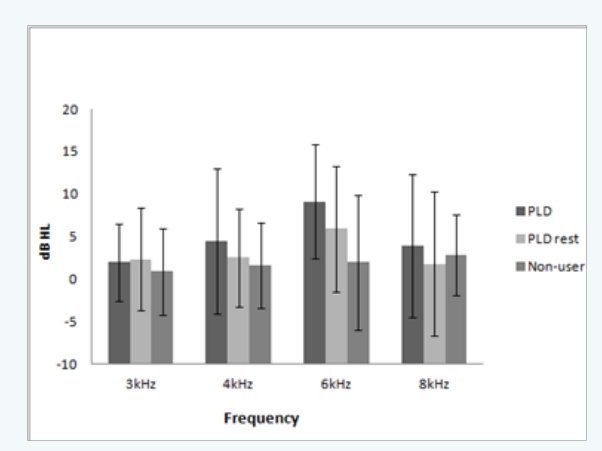

Figure 4: The mean high frequency hearing threshold across 3 $\mathrm{kHz}$ to $8 \mathrm{kHz}$ frequencies in the left ear for PLD-user, PLD-rest and Non-user.

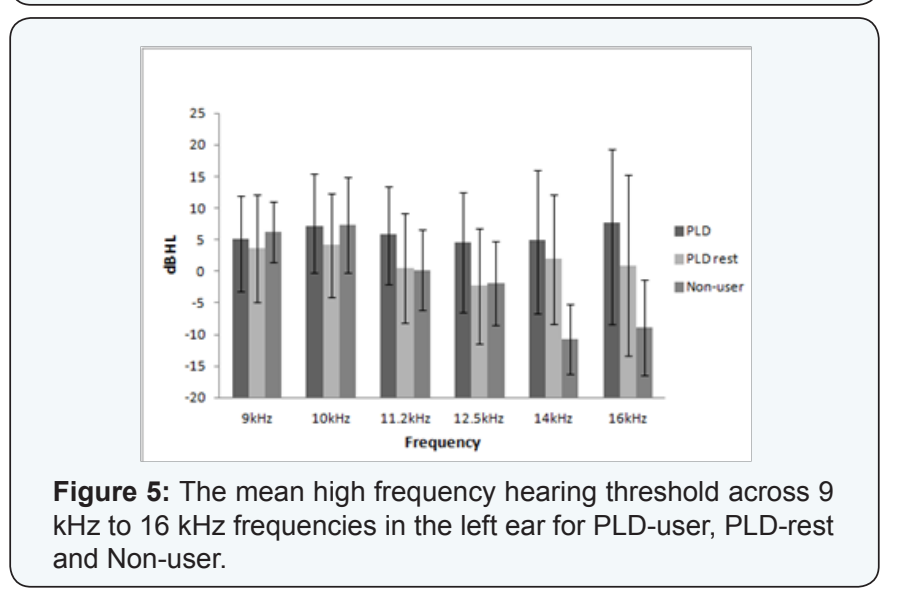


Mann Whitney U test showed that PLD user group had significantly poorer hearing thresholds at $11.2 \mathrm{kHz}, 12.5 \mathrm{kHz}$, $14 \mathrm{kHz}, 16 \mathrm{kHz}$ and $18 \mathrm{kHz}$ in both ears. Furthermore, PLD user group had significantly poor hearing thresholds at $4 \mathrm{kHz}, 6 \mathrm{kHz}$ in the right ear. Mann Whitney $U$ test revealed that there was no statistically significant difference in hearing thresholds between PLD-user and PLD-rest group for all frequencies tested in both the ears. These results indicate that rest period of 15 days did not change the hearing thresholds of PLD use.

\section{Transient evoked otoacoustic emissions (TEOAEs)}

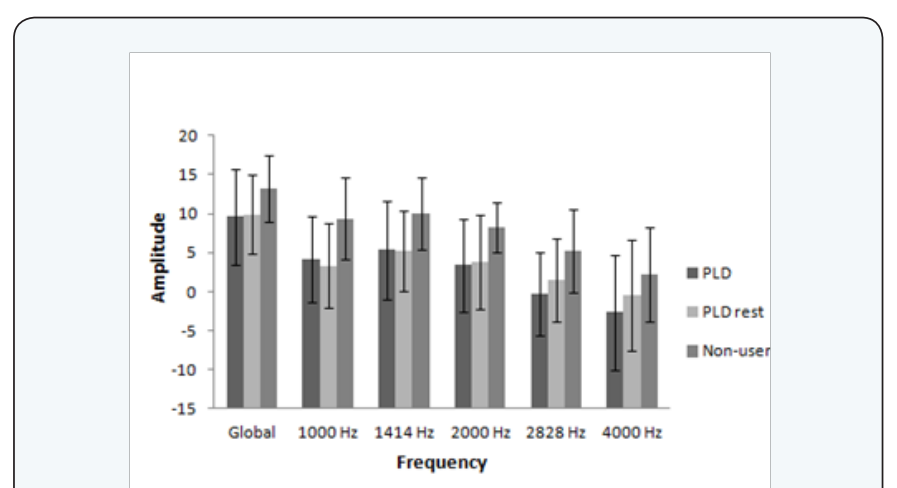

Figure 6: TEOAE amplitude across different frequencies in the right ear for PLD-user, PLD-rest and Non-user.

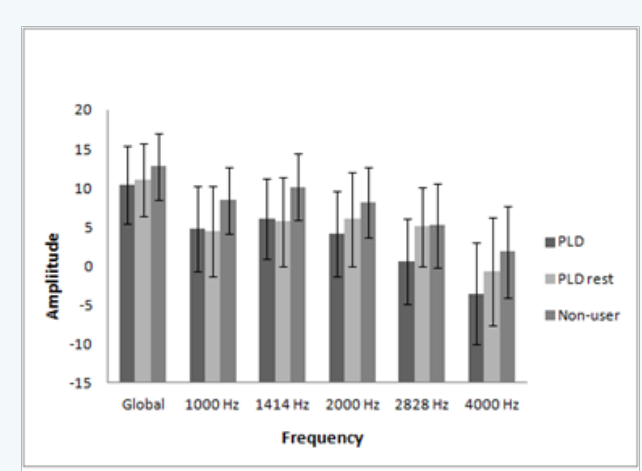

Figure 7: TEOAE amplitude across different frequencies in the left ear for PLD-user, PLD-rest and Non-user.

Figures 6 \& 7 shows mean TEOAE amplitudes in three groups in right and left ear along with one standard deviation of error. Both the overall and band wise TEOAE amplitudes are shown in (Figure 3a \& 3b). From (Figures 3a \& 3b) it can be seen that both overall and band wise TEOAE amplitudes were reduced in PLDgroup and PLD-rest group compared to Non-user group. Since standard deviations of the otoacoustic emission amplitudes were high non parametric test was used to evaluate the significance of difference among hearing thresholds in PLD user, PLD rest and PLD non user group. Mann Whitne U-test revealed that TEOAE amplitudes were significantly reduced in PLD user group compared to non PLD user at all the frequencies except for the left ear at global amplitude $(Z=-1.554, p>0.05)$. However, there was no statistically significant difference between PLD- user and
PLD-rest for both the ears at all frequency.

\section{Speech Perception in Noise}

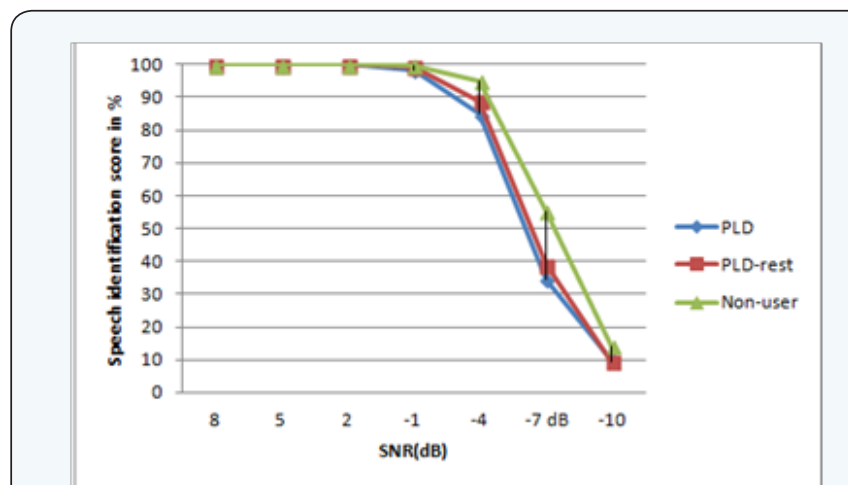

Figure 8: The mean percentage for PLD-users, PLD-rest and Non-users across different SNR levels.

(Figure 8) shows mean word identification scores at different signal to noise rations (SNR) in three groups. From the above data threshold SNR required to obtain the $50 \%$ speech identification scores were calculated using Spearman and Karber equation (Finney, 1952).

$50 \%=\mathrm{i}+(\mathrm{d})(\mathrm{d})(\#$ correct $)=(\mathrm{w})$

Where $\mathrm{i}=$ the initial presentation level $(\mathrm{dB} \mathrm{S} / \mathrm{N})$,

$\mathrm{d}=$ the attenuation step size (decrement),

$\mathrm{w}=$ the number of items per decrement.

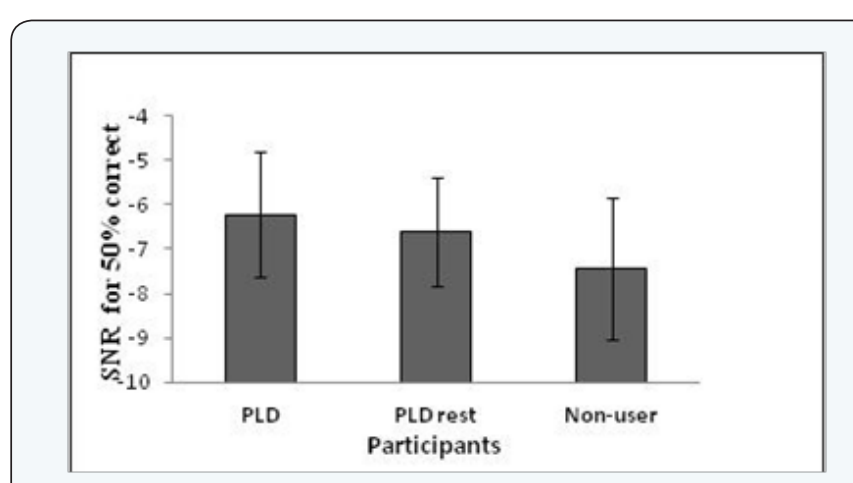

Figure 9: Average SNR-50 for the PLD-user, PLD-rest and Nonuser group.

(Figure 9) shows mean SNR-50 for the PLDs-group, PLD-rest group and the Non-user group along with one standard deviation of error. Mann-Whitney U test was used to find the significance of difference in SNR-50 obtained among three groups. Results revealed that Non-user group had lower SNR-50 compared to other two groups $(\mathrm{Z}=-2.891, \mathrm{p}<0.05$ [PLD-user and non-user] and $\mathrm{Z}=-2.174, \mathrm{p}<0.05$ [PLD-rest and non-user]). However, there was no significant difference was noticed between PLD-user and PLD-rest group. These results suggest that use of PLDs results in poorer SNR-50 and rest period of 15 will not improve the condition. 


\section{Global Journal of Otolaryngology}

\section{Discussion}

The purpose of this study was to measure the output levels of personal listening devices (PLDs) at the volume control setting that was preferred by the subjects in quiet, in the presence of bus noise and at the maximum output level of the devices. Furthermore, this study also evaluated the extended high frequency hearing thresholds, otoacoustic emission and speech perception skills in the group of individuals who uses PLDs and compared that to same individuals after 15 days of period abstaining from music listening and to individuals who did not use PLDs. The average output sound pressure level in quiet condition was noted to be $73.34 \mathrm{dBA}$ (Range 51.98 to 99.54 $\mathrm{dBA}$ ). These preferred listening levels are quite similar to what participants selected as "sounds best to you" in the 2007 [1], or "medium/comfortable" in the 2008 [10]. In the presence of bus noise the average output level went up to $79.44 \mathrm{dBA}$ (Range 60.02 to $112.80 \mathrm{dBA}$ ).

This increase in output levels in presence of background noise is comparable to 2007 [1]. Similar findings were also obtained in the study by Heines, Hodgetts, Ostevik and Reiger (2012), who reported that the average output levels of iPods in quiet condition, was $70.3 \mathrm{dBA}$ and in the presence of transportation noise was $83.5 \mathrm{dBA}$. But the measured output level by in presence of bus noise is slightly higher than what is obtained in the present study. This might be due to the different types of ear phones used in the study. In the present study along with the ear-bud type ear phones other ear phones like half concha, supraaural earphone are also used only the ear bud type of ear phones. This might have resulted in higher ear canal output sound pressure levels because of reduced leakage of sound. No evidence based definition exists for hazardous sound levels of music.

As a substitute, standards for exposure to occupational noise have been proposed for use. In India, the Ministry of Environment Forest [11] has proposed a time weighted average level of 80 $\mathrm{dBA}$ for an 8-hour period per day as the maximum permissible limit. '5 dB exchange rule' has been proposed by the Ministry of Environment and Forests as a tradeoff between the expo-sure time and sound level. Considering this criteria output levels of PLDs at maximum volume control settings or in presence of bus noise is high and may result in permanent noise induced hearing loss if exposed for extended periods of time (years).

\section{Effect of PLDs on auditory measure}

Results of the extended high frequency pure tone audiometry showed that hearing thresholds of individuals who used PLDs are significantly poorer compared to Non-users. Results of the present study are in agreement with [12]. She reported that hearing thresholds in the extended high frequency regions were significantly poor in individuals who used PLDs compared to individuals who did not use. Extended high frequency hearing thresholds are reported to be more sensitive to noise induced damages than the conventional audiometric frequencies. Peng et al. [7] reported that extended high frequencies may be affected by the noise earlier when compared to conventional audiometric frequencies.

Transient otoacoustics emission (TEOAE) amplitudes were significantly poorer in PLDs-user and PLDs-rest group compared to Non-user. Kumar et al. [6] reported a negative correlation between DPOAE amplitudes and output SPLs of PLDs at preferred volume control settings. They concluded that individuals who listened to music at higher levels had reduced DPOAE amplitudes even though the DPOAE amplitudes were within the clinical norms. Reported reduced TEOAE amplitude in the individuals using PLDs compared to individuals who don't use. Miller, Marshall, Heller and Hughes (2006) [13] reported that amplitudes of DPOAEs are more sensitive to noise induced hearing loss than pure tone hearing thresh-olds. Barros et al. [12] suggested that TEOAEs are sensitive tool to identify temporary alteration in cochlea produced by exposure to an elevated sound pressure level. Individuals who used PLDs had significantly poor speech perception abilities in noise compared to Non-user. The observed deterioration in the speech processing skills in the PLD users, probably due to changes in the central auditory system caused due to prolonged exposure to loud mu-sic. It has been reported that long-term noise may have a persistent effect on brain function and behavior, even when the peripheral hearing sensitivity is within normal range [14].

None of the auditory measures-extended high frequency audiometry, TEOAE or speech perception in noise - changed significantly following 15 days of abstaining from music. These results suggest that listening to high levels of music through PLDs causes 'permanent damage both peripheral and central auditory structures. Kujawa \& Brattico [15] reported a rapid and irreversible degeneration of spiral ganglion cells by the noise exposure which resulted in temporary threshold shifts. Even after, hair cells and hearing sensitivity were recovered, neuronal loss persisted. The effects of such neuronal losses on auditory and speech processing are detriment

\section{Summary and Conclusion}

Hearing loss induced by personal listening devices (PLDs) may evolve into a significant social and public health problem in future years. Previous studies have shown that out-put levels of PLDs can be as high as $113 \mathrm{dBA}$. Prolonged exposure to loud music leads to a significant/subclinical damage to hair cells of the cochlea. This in turn may lead to dysfunction in the central auditory system. Hence the current study was taken up with the following objectives:

i. To measure the output levels of PLDs at the volume control setting that was preferred by the subject in quiet and in the presence of $65 \mathrm{~dB}$ SPL bus noise and at the maximum volume setting of devices. 
ii. To compare the transient evoked otoacoustic emissions (TOAEs) in individuals who use PLDs and individuals after abstaining from listening to music through PLDs.

iii. To compare the extended high frequency hearing thresholds ( $3 \mathrm{kHz}-20 \mathrm{kHz}$ ) in individuals who use PLDs and individuals abstaining from listening to music through PLDs.

iv. To measure speech perception in noise in individuals who use PLDs and individuals abstaining from listening to music through PLDs.

\section{Following the results were obtained in the present study}

a. The mean $\mathrm{dBA}$ at preferred volume control settings in quiet was $73 \mathrm{dBA}$. In the presence of bus noise of $65 \mathrm{~dB}$ SPL the mean preferred listening levels were increased to $79 \mathrm{dBA}$ and at the maximum output level of the devices was $88 \mathrm{dBA}$.

b. Extended high frequency hearing thresholds, amplitudes of TEOAEs and SNR-50 values were significantly poor in individuals who used PLDs compared individuals who did not use PLDs.

c. None of the auditory measures - extended high frequency audiometry, TEOAE or speech perception in noise - changed significantly following 15 days of abstaining from music. These results suggest that listening to high levels of music through PLDs causes' permanent damage both peripheral and central auditory structures. From the above results, it can be concluded individuals who listen to music through PLDs may be putting themselves at risk for permanent noise induced hearing loss if exposed for extended periods of time (years). These results are alarming as more and more children are using PLDs. Some of the recommendations that should be kept in mind while us-ing PLDs are:

1) Not to keep volume control of the device very high.

2) To take periodic breaks of 15-20 minutes when listening to music.

3) Use loose-fitting ear buds or headphones to minimize intensity of sound.

4) Device should have an alarming indication when it reached to a hazardous levels

\section{References}

1. Hodgetts WE, Rieger JM, Szarko RA (2007) The effects of listening environment and earphone style on preferred listening levels of normal hearing adults using an mp3 player. Ear Hear 28(3): 290-297.

2. Fligor BJ, Cox LC (2004) Output levels of commercially available portable compact disc players and the potential risk to hearing. Ear Hear 25(6): 513-527.

3. Catalano PJ, Levin SM (1985) Noise induced hearing loss and portable radios with headphones. Int J Pediatr Otorhinolaryngol 9(1): 59-67.

4. Karen Zaums, Donald Fucci, Molly Banks, Linda Petrosino, Corry Wilcox (1974) Listening patterns of self-identified rock music presented via earphones. Journal of Auditory Research 14: 51-56.

5. Williams W (2005) Noise exposure levels from personal stereo use. Int J Audiol 44(4): 231-236.

6. Kumar A, Mathew K, Alexander SA, Kiran C (2009) Volume output sound pressure levels of personal music systems and their effect on hearing. Noise Health 11(44): 132-140.

7. Peng JH, Tao ZZ, Huang ZW (2007) Risk of damage to hearing from personal listening devices in young adults. J Otolaryngol 36(3): 181185.

8. Raymond Carhart, James F Jerger (1959) Preferred method for clinical determination of pure-tone thresholds. The Journal of Speech and Hearing Disorders 24: 330-345.

9. Methi RA \& Kumar, UA (2009) Development of sentence material for quick speech in noise test (quick sin) in kannada. Journal of Indian speech and Hearing Association 23(1): 59-65.

10. Torre $P 3^{\text {rd }}(2008)$ Young adults use and output level set-tings of personal music systems. Ear Hear 29(5): 791-799.

11. Ministry of Environment and Forest (2000) Noise pollution (regulation control). Technical Report S. O. 123 (E), Ministry of Environment and Forest.

12. Barros SM, Frota S, Atherino CC, Osterne F (2007) The efficiency of otoacustic emissions and pure tone audiometry in the detection of temporary auditory changes after exposure to high sound pressure levels. Braz J Otorhinolaryngol 73(5): 592-598.

13. Vogel I, Brug J, van der Ploeg CP, Raat H (2007) Young People's exposure to loud music, a summary of the literature. Am J Prev Med 33(2): 124-133.

14. Kujawa SG, Liberman MC (2009) Adding insult to injury: Cochlear nerve degeneration after temporary noise-induced hearing loss. J Neurosci 29(45): 14077-14085.

15. Kujala T, Brattico E (2009) Detrimental noise effects on brain's speech functions. Biol Psychol 81(3): 135-143. 